\title{
Evolution of signatures of quasifission in reactions forming curium
}

\author{
E. Williams, ${ }^{*}$ D. J. Hinde, M. Dasgupta, R. du Rietz, ${ }^{\dagger}$ I. P. Carter, M. Evers, D. H. Luong, \\ S. D. McNeil, D. C. Rafferty, K. Ramachandran, ${ }^{\ddagger}$ and A. Wakhle \\ Department of Nuclear Physics, Research School of Physical Sciences and Engineering, \\ The Australian National University, Canberra, ACT 0200, Australia
}

(Received 29 July 2013; published 25 September 2013)

\begin{abstract}
Background: Quasifission, a fission-like reaction outcome in which no compound nucleus forms, is an important competitor to fusion in reactions leading to superheavy elements. The precise mechanisms driving the competition between quasifission and fusion are not well understood.

Purpose: To understand the influence reaction parameters have on quasifission probabilities, an investigation into the evolution of quasifission signatures as a function of entrance channel parameters is required.

Methods: Using the Australian National University's (ANU) CUBE detector for two-body fission studies, measurements were made for a wide range of reactions forming isotopes of curium. Important quasifission signatures-namely, mass-ratio spectra, mass-angle distributions, and angular anisotropies-were extracted.

Results: Evidence of quasifission was observed in all reactions, even for those using the lightest projectile $\left({ }^{12} \mathrm{C}+{ }^{232} \mathrm{Th}\right)$. But the observables showing evidence of quasifission were not the same for all reactions. In all cases, mass distributions provided some evidence of the possible presence of quasifission but were not sufficient in most cases to clearly identify reactions for which quasifission was important. For reactions using light projectiles $\left({ }^{12} \mathrm{C},{ }^{28,30} \mathrm{Si},{ }^{32} \mathrm{~S}\right)$, experimental angular anisotropies provided the clearest signature of quasifission. For reactions using heavier projectiles $\left({ }^{48} \mathrm{Ti},{ }^{64} \mathrm{Ni}\right)$, the presence of mass-angle correlations in the mass-angle distributions provided strong evidence of quasifission and also provided information about quasifission timescales.

Conclusions: The observable offering the clearest signature of quasifission differs depending on the reaction timescale.
\end{abstract}

DOI: $10.1103 /$ PhysRevC.88.034611

PACS number(s): 24.75.+i, 25.85.-w, 25.70.Jj

\section{INTRODUCTION}

The "island of stability" is an area of the nuclear chart thought to contain long-lived superheavy elements due to predicted shell closures near proton number 114 and neutron number 184 [1]. This island-or more particularly, the superheavy elements it includes-has been an ongoing quest in the field of nuclear physics since the 1960s [1-3]. The boundaries of the island, and the properties of the isotopes it contains, provide extremely sensitive tests of our understanding of nuclear shell structure. To date, superheavy elements up to $Z=118$ have successfully been made using either "cold" $[3,4]$ or "hot" fusion [5]. But forming each new superheavy element has been a significant experimental challenge and has only allowed access to the neutron-poor side of the island. With cross sections on the order of picobarns $[3,4,6,7]$ and the need for increasingly neutron-rich beams, only a new level of understanding of nuclear reactions will allow us to map out possibilities for exploring deeper within the island's boundaries.

In reactions between heavy nuclei, capture is the first step towards nuclear fusion. In capture, the system loses its relative kinetic energy and may be trapped within the pocket in the entrance-channel potential. It then undergoes shape evolution, in which the dinuclear system may evolve

\footnotetext{
${ }^{*}$ Corresponding author: elizabeth.williams@anu.edu.au

†Current address: Malmö University, Malmö 205 06, Sweden.

${ }^{\ddagger}$ Permanent address: Nuclear Physics Division, Bhabha Atomic Research Centre, Mumbai 400085, India.
}

towards a mononuclear shape. At this point, the system can follow two paths: (1) form a compact compound nucleus, and therefore fuse, or (2) split apart again before reaching a compact equilibrium shape. This second process is known as quasifission and is the most important competitor to fusion in reactions capable of forming superheavy elements.

The suppression of compound-nucleus formation due to quasifission has several consequences. Reactions dominated by quasifission occur faster (by as much as four orders of magnitude) than those dominated by fusion-fission $[8,9]$ and can be strongly influenced by entrance channel variables $[8,10-12]$. Quasifission outcomes have also been associated with a reduction in evaporation residue cross sections [13]. Nevertheless, an understanding of how all signatures of quasifission evolve as a function of reaction timescales is not yet clear.

Quasifission is difficult to study from an experimental perspective because it can appear very much like fusion-fission. Individual fission events resulting from quasifission can be indistinguishable in all characteristics from their fusion-fission counterparts. But in the case of quasifission, the elongated projectile-target system never reaches the equilibrium shape required in fusion-fission. Instead, the colliding nuclei stick together long enough for the masses of the projectile- and target-like components to evolve towards symmetry before coming apart again. Within the distributions of fission fragment characteristics, then, some signatures of this dynamic collision process should remain.

In this work, different signatures of quasifission will be presented for a range of reactions leading to isotopes of curium. In studying these reactions, the relationship between different 
signatures of quasifission will be explored. By seeking out when and how quasifission signatures appear, and exploring what these signatures mean in terms of the physics driving quasifission outcomes, we aim to better understand precisely where the limitations in our understanding of quasifission lie. In the process, we provide a range of experimental results that can be used to benchmark current theory and trigger new developments in dynamical reaction models.

\section{EXPERIMENTAL SIGNATURES OF QUASIFISSION}

Evidence of quasifission can be found in a variety of observables, including angular distributions, mass distributions, and mass-angle distributions. The relationship between each of these observables and quasifission will be discussed briefly here, in order to provide context for this exploration of quasifission in reactions leading to curium.

\section{A. Mass distributions}

Mass distributions of two-body fission fragments were the first observable associated with a nonequilibrium fission process [14]. In particular, the width of the mass distribution can be used as a tool for identifying the presence of quasifission $[15,16]$.

In simple models of fusion-fission, all degrees of freedom (including mass) are assumed to achieve equilibrium statistical values, which are typically associated with the fission saddle point. For mass distributions peaked at symmetry, the variance of the mass distribution for such systems is expected to behave as follows:

$$
\sigma_{M}^{2}=v T+\mu\left\langle L^{2}\right\rangle,
$$

where $T$ is the temperature of the compound nucleus at the saddle point, $\left\langle L^{2}\right\rangle \equiv\left\langle\ell^{2}\right\rangle \hbar^{2}$ is the mean-squared angular momentum, and $\mu, v$ are parameters [14,17-19]. The widths of such mass distributions are typically narrow, although precisely what "narrow" means is poorly defined in the literature. For now, only a comparative definition can be made: for reactions leading to a given compound nucleus, equivalent reactions dominated by quasifission may exhibit wider mass distributions than those dominated by fusionfission $[8,15,20,21]$. A model capable of accurately predicting both the fusion-fission and quasifission mass distributions is an important goal for the field.

Several mechanisms are responsible for the increased width observed in quasifission reactions. The first mechanism is directly related to how long the projectile-target system sticks together before coming apart again. This "sticking time" has consequences for the amount of mass equilibration between the projectile- and target-like nuclei as well as the kinematics of the observed fission fragments. These consequences, which result in a nonisotropic distribution of fragment mass as a function of reaction angle, will be discussed in Sec. (II C) below. Sticking time may also have additional effects on mass distributions as a result of the relationship between the variance of the mass distribution and temperature given in Eq. (1). For fast nonequilibrium fission processes, the system has less time to cool itself via particle evaporation; a higher temperature and, consequently, a wider mass distribution can result. The second mechanism is related to the diverse paths nonequilibrium projectile-target systems can take to scission. The broad definition of quasifission taken in this work (a definition chosen to be consistent with what we are able to measure experimentally) means that many different nonequilibrium processes can contribute to the observed mass distribution. Some collisions may be equilibrated in all but elongation [22]; at the other extreme, others may not even have overcome the saddle point on their path to scission.

\section{B. Angular distributions and anisotropies}

Angular distributions of fission fragments, or $W(\theta) \equiv$ $d \sigma(\theta) / d \Omega$, with $\theta$ being the angle relative to the beam axis, have long been used to identify the presence of quasifission [23-25] in heavy-ion reactions. In particular, angular anisotropies, given by $A \equiv W\left(0^{\circ}\right) / W\left(90^{\circ}\right)$ or $W\left(180^{\circ}\right) / W\left(90^{\circ}\right)$, are a key signature in identifying the presence of quasifission. To understand how anisotropies can be used to identify quasifission, a brief review of how anisotropies behave in the standard transition-state model (TSM) [26,27] is required.

In the TSM, fission is taken to occur in the direction of the nuclear symmetry axis at the fission saddle point. An approximate expression for the calculated anisotropy is given by [26]

$$
A \sim 1+\frac{\left\langle L^{2}\right\rangle}{4 K_{0}^{2}}=1+\frac{\left\langle\ell^{2}\right\rangle \hbar^{2}}{4 T \mathcal{J}_{\text {eff }}},
$$

where $\left\langle L^{2}\right\rangle$ is the mean squared angular momentum, $T$ is the temperature, and $\mathcal{J}_{\text {eff }}$ is the effective moment of inertia of the nucleus at the saddle-point deformation. Reasonable agreement between expectations based on the TSM and experiments has been found in reactions where quasifission is not expected, such as ${ }^{16} \mathrm{O}+{ }^{208} \mathrm{~Pb}$ [27]. In cases where experimentally observed anisotropies are significantly larger than the TSM predictions, quasifission (or, to use another nomenclature, nonequilibrium fission) has been attributed as the cause [23-25]. However, no dynamic models of angular distributions are in common use, making quantitative interpretations of these large anisotropies challenging.

\section{Mass-angle distributions}

The most direct evidence of the existence of quasifission can be found in the mass-angle distributions (MADs), which are the distribution of fission-fragment yield as a function of mass or mass ratio and center-of-mass angle $\theta_{\text {c.m. }}$. The anisotropies and mass ratios discussed above are subsets of MADs, as mass ratios are simply a MAD projected over all angles, and anisotropies are extracted from angular distributions, which are proportional to the projection onto $\theta_{\text {c.m. }}$. of MADs for all masses.

Evidence of quasifission appears in the form of a correlation between the mass ratio distribution and $\theta_{\text {c.m. }}$. This correlation is directly related to the amount of time the projectile- and 
target-like nuclei remain in contact before coming apart; thus, the MAD provides direct information on the timescale of quasifission [8].

The presence of a mass-angle correlation can be understood if one considers the consequences that a relatively short period of contact, or sticking time, between the projectile- and targetlike nuclei will have. The sticking time for a given reaction is approximately given by

$$
\tau=\frac{\Delta \theta}{\langle\omega\rangle},
$$

where $\langle\omega\rangle$ is the average angular velocity during the collision and $\Delta \theta$ is the angle of rotation of the system during the reaction. From Tōke et al. [8], the mass drift between the two systems is thought to begin approximately $2 \times 10^{-21}$ seconds after the reaction begins, and then proceed asymptotically towards symmetry $\left(M_{R}=0.5\right)$ with a mass-equilibration time constant of $\sim 5.2 \times 10^{-21}$ seconds. As illustrated in Ref. [9], these simple ingredients, assuming a parabolic potential, a constant moment of inertia, angular momentum distributions for capture, and classical Coulomb trajectories for incoming and outgoing nuclei, provide a means of simulating the effect different sticking times have on mass-angle correlations.

The picture presented by Tōke et al. [8] and expanded to calculate MADs by du Rietz et al. [9] has not been tested for a wide range of reactions. However, the interpretation suggests two things: (1) fission fragments approaching the equilibrium $M_{R}$ of 0.5 are most likely to be formed in reactions with longer sticking times, and (2) if the two systems undergo less than half a rotation before scission, the average angle between the projectile- and target-like fragments will bear some trace of the projectile's incoming trajectory. As a result, one can use a simple mass-transfer model and basic kinematics to extract information about the timescale of quasifission reactions for which a mass-angle correlation is observed.

Since fusion-fission cannot give rise to a mass-angle correlation, the observation of such a correlation provides direct evidence of quasifission. As such, MADs provide striking evidence for the presence of quasifission and, with their direct dependence on sticking times, also provide invaluable insight into the timescale and detailed dynamics of quasifission.

\section{Additional signatures}

There are a wide range of additional techniques used to study quasifission. The clearest additional observable relating to quasifission is the measurement of evaporation residue (ER) yields; evidence of fusion suppression in heavy systems has been attributed to quasifission [13]. However, in many of these heavy-ion reactions, the ER cross sections are very small, making this a particularly challenging choice for studies of fusion hindrance caused by competition from quasifission.

Other methods used to study quasifission include the observations of prescission neutron multiplicities [28-30], crystal blocking [31-33], and x-ray measurements [34]. Prescission neutrons have been used as a "neutron clock" to study quasifission, but all this work, to date, has resulted in conflicting conclusions [28-30,35]. Crystal blocking measurements, also sensitive to the timescale of a fission process, yield timescales for fission reactions that are much longer than those predicted for the same reactions using MADs [35]. X-ray measurements, which are just beginning to be used to study compound-nucleus formation in reactions with a high fission probability, have only been carried out for a small selection of reactions [34]. In all these signatures, interpretations of quasifission properties are challenging at best and contradictory at worst, providing further incentive to explore quasifission in a wide range of systems using all available means.

This work uses some of the most accessible observablesmass ratios, MADs, and anisotropies-to explore what observed fission fragments can tell us about quasifission. If one signature of quasifission is observed, does this mean all observables will exhibit some sign of this nonequilibrium process? If not, then what are the relationships between these signatures, and how can we use them to get a more detailed picture of how quasifission evolves as a function of entrance channel parameters?

\section{EXPERIMENTAL METHODS}

In order to study how quasifission signatures evolve as a function of entrance-channel parameters for reactions leading to curium, a series of experiments were performed at the Heavy Ion Accelerator Facility at the Australian National University (ANU). All studied reactions are listed in Table I, along with beam energies, target properties, etc. In all cases, the 14UD tandem accelerator was used to produce a pulsed beam of projectiles (pulse width $\sim 1 \mathrm{~ns}$, pulse separation $\sim 106 \mathrm{~ns}$ ). In the case of the heaviest projectile, the ANU superconducting linear accelerator was used to boost the beam energy to energies above the Coulomb barrier.

Fission fragments resulting from each reaction were observed using the ANU CUBE detector system [36], which consists of two large-area position-sensitive Multiwire Proportional Counters (MWPCs). Two Si monitor detectors at forward angles, located symmetrically about the beam axis, are used for absolute cross section normalization. Two different CUBE configurations were used for most of the selected reactions. As shown in Fig. 1(a), the $\theta=135^{\circ}$ configuration was used for angular distribution (anisotropy) measurements in order to ensure coverage towards $180^{\circ}$. In all but the case of the lightest projectile, a $\theta=90^{\circ}$ configuration as shown in Fig. 1(b) was used to measure mass-angle distributions (MADs). For both configurations, the angular coverage is demonstrated in the MAD plots in Figs. 1(c) and 1(d), respectively.

Kinematic reconstruction of all events was based on twobody kinematics using the methods outlined in Refs. [15,36]. From position and timing information, as well as the known geometry of the setup, fission-fragment velocities and centerof-mass angles were reconstructed. Mass ratios, given by

$$
M_{R}=\frac{M_{1}}{M_{1}+M_{2}},
$$

were also determined. The total parallel and perpendicular velocities of each coincident pair of fission fragments were also calculated and compared to prediction for two-body full momentum transfer (FMT) fission, as explained in Ref. [37]. 
TABLE I. Summary of reaction parameters for all experiments presented in this work.

\begin{tabular}{|c|c|c|c|c|c|}
\hline${ }^{12} \mathrm{C}$ & ${ }^{232} \mathrm{Th}\left(300 \mu \mathrm{g} / \mathrm{cm}^{2}\right)$ & ${ }^{27} \mathrm{Al}\left(30 \mu \mathrm{g} / \mathrm{cm}^{2}\right)$ & ${ }^{244} \mathrm{Cm}$ & $61-94$ & $58-90$ \\
\hline${ }^{30} \mathrm{Si}$ & ${ }^{208} \mathrm{PbS}\left(40 \mu \mathrm{g} / \mathrm{cm}^{2}\right)$ & ${ }^{\text {nat }} \mathrm{C}\left(12 \mu \mathrm{g} / \mathrm{cm}^{2}\right)$ & ${ }^{238} \mathrm{Cm}$ & $154-167$ & $135-146$ \\
\hline${ }^{28} \mathrm{Si}$ & ${ }^{208} \mathrm{PbS}\left(90 \mu \mathrm{g} / \mathrm{cm}^{2}\right)$ & ${ }^{n a t} \mathrm{C}\left(10 \mu \mathrm{g} / \mathrm{cm}^{2}\right)$ & ${ }^{236} \mathrm{Cm}$ & $138-188$ & $120-164$ \\
\hline${ }^{30} \mathrm{Si}$ & ${ }^{206} \mathrm{PbS}\left(100 \mu \mathrm{g} / \mathrm{cm}^{2}\right)$ & ${ }^{\text {nat }} \mathrm{C}\left(10 \mu \mathrm{g} / \mathrm{cm}^{2}\right)$ & ${ }^{236} \mathrm{Cm}$ & $135-198$ & $119-175$ \\
\hline${ }^{32} \mathrm{~S}$ & ${ }^{202} \mathrm{HgS}\left(80 \mu \mathrm{g} / \mathrm{cm}^{2}\right)$ & ${ }^{\text {nat }} \mathrm{C}\left(15 \mu \mathrm{g} / \mathrm{cm}^{2}\right)$ & ${ }^{234} \mathrm{Cm}$ & $157-191$ & $136-165$ \\
\hline${ }^{64} \mathrm{Ni}$ & ${ }^{170} \mathrm{Er}_{2} \mathrm{O}_{3}\left(96 \mu \mathrm{g} / \mathrm{cm}^{2}\right)$ & ${ }^{\text {nat }} \mathrm{C}\left(30 \mu \mathrm{g} / \mathrm{cm}^{2}\right)$ & ${ }^{234} \mathrm{Cm}$ & $280-318$ & $204-231$ \\
\hline
\end{tabular}

Events consistent with FMT fission were selected based on this parallel and perpendicular velocity reconstruction. Further gate selections based on $M_{R}$ and total kinetic energy (TKE) could also be made in order to isolate fission or elastic scattering events, as required.

Absolute cross sections and angular distributions were also determined following a similar kinematic analysis by comparing to a calibration run taken with the same CUBE configuration using a low-energy reaction in which only elastic scattering is possible [27]. Anisotropies were determined from a fit of the observed angular distributions using FIFRANG, an in-house fission fragment angular distribution fitting code [38].

\section{THEORETICAL METHODS}

Before presenting the experimental results, a discussion of the theoretical methods used to interpret these results is
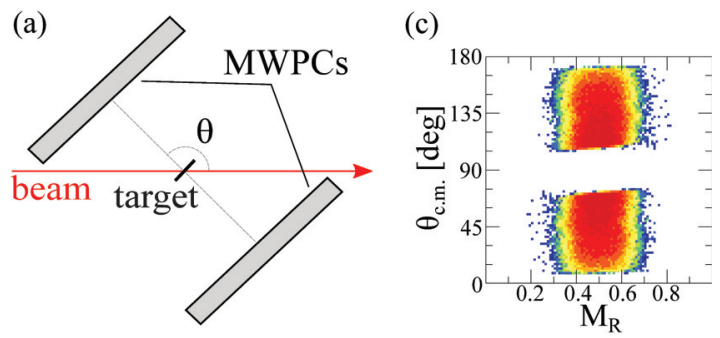

(b)

(d)
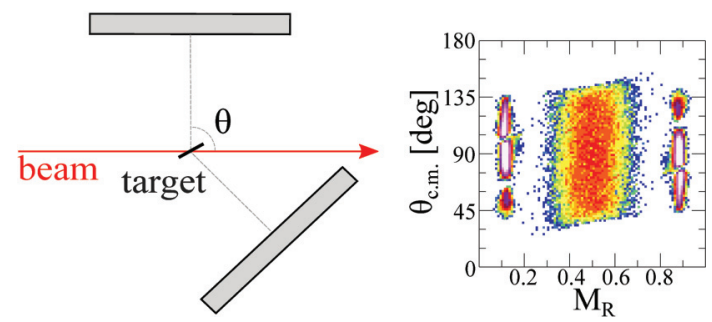

FIG. 1. (Color online) The ANU CUBE spectrometer's Multiwire Proportional Counters can be configured in two ways: with the back detector at (a) $\theta=135^{\circ}$, which provides better angular coverage for anisotropy measurements and reactions using light projectiles, and (b) $\theta=90^{\circ}$, which is preferred for mass-angle distribution measurements and reactions with heavier projectiles. The angular coverages (mirrored across both $\theta_{\text {c.m. }}=90^{\circ}$ and $M_{R}=0.5$ ) for the $\theta=135^{\circ}$ and $\theta=90^{\circ}$ configurations, respectively, are illustrated using mass-angle distributions in panels (c) and (d). required. Because quasifission distributions overlap with those from fusion-fission, arguments supporting the presence of quasifission are typically based on comparison to standard fusion-fission models, such as the TSM.

The TSM calculations, in turn, require inputs such as the $\ell$ distributions following fusion. The $\ell$ distributions following fusion-fission can be obtained from a coupled-channels fusion model as described in the next section.

\section{A. Coupled-channels model}

For the anisotropy calculations presented in this work, partial wave distributions ( $\ell$ distributions) were calculated for all reactions using either CCFULL $[39,40]$ or CCDEGEN, a variant of CCFULL for rotational target nuclei.

Finding a consistent way to carry out these coupledchannels calculations for the wide range of reactions presented here was a challenge. Many different projectile and target structures were chosen for this work, and experimental barrier distribution data-which are helpful for determining the average barrier and identifying the nature of the couplings involved-were not available in most cases. In order to reduce the parameter space of the model and provide a systematic approach to calculations for all reactions studied in this work, the incoming wave boundary condition (IWBC) was used. The methods we used to select both the Woods-Saxon potential and nuclear structure parameters are described here.

\section{Nuclear potential parameters}

In CCFULL and CCDGEN, a Woods-Saxon (WS) form of the nuclear potential is used:

$$
V_{N}(r)=\frac{V_{0}}{1+\exp \left[\left(r-R_{0}\right) / a\right]},
$$

where $V_{0}$ is the depth of the potential in $\mathrm{MeV}, a$ is its diffuseness in units of femtometers, and $R_{0}=r_{0}\left(A_{p}^{1 / 3}+A_{t}^{1 / 3}\right) . A_{p}$ and $A_{t}$ refer to the projectile and target masses, respectively, while $r_{0}$ is a parameter in units of femtometers.

The parameters of this potential, $V_{0}, a$, and $r_{0}$, all have a significant and somewhat interdependent influence on the calculation outcome, particularly for above-barrier energies. Because of this, they must be chosen rather carefully, and the calculation output must be checked for evidence of nonphysical behavior. In particular, a decrease in the above-barrier cross section as a function of energy is a sign that the potential 
pocket is not sufficiently deep. Rapid, oscillating changes in the calculated barrier distribution at high energies are a sign that reflections out of the potential pocket are occurring. In either scenario, all calculation outputs-including the $\ell$ distributions of interest-bear signs of the model's failure in the higher-energy results.

To add to the complexity of these calculations, it is well known that diffuseness parameters $a$ required to reproduce fusion cross-section data can vary considerably, depending on the reaction in question [43]. Although a value of $a \sim 0.65$ is required to reproduce elastic and quasielastic scattering data [44-46], Newton et al. found that values higher than $a \sim 1.2$ are needed to reproduce fusion data, possibly reflecting the model's failure to account for the likely dissipative processes underlying fusion. Therefore, all TSM calculations in this work were done using CCFULL calculations for a range of diffuseness parameters, $a=0.65,1.0$, and 1.08 or $1.2 \mathrm{fm}$, in order to check the sensitivity of the anisotropy calculations to the diffuseness parameter.

For all calculations, a simple approach to selecting model parameters was used. For each diffuseness parameter, $r_{0}$ was considered fixed, and $V_{0}$ was adjusted until the Swiatecki [42] or, for lighter projectile nuclei, Sao Paulo [41] barrier energy $\left(V_{B}\right)$ was reproduced for the uncoupled (structure-free) calculation. Nuclear structure coupling (discussed below) was then added in, and $V_{0}$ was once again adjusted to reproduce the average barrier energy. The final $V_{0}$ values used in the calculations that included coupling are given in Table II.

All WS parameter choices used in this work are provided in Table II. From this table, one can see that a careful balance was required to ensure physically realistic calculations when using the incoming wave boundary condition. While every attempt was made to fix $r_{0}$ to the same value for all calculations, it quickly became clear that this was not possible for the wide range of reactions of interest. As the diffuseness parameter was increased, smaller $r_{0}$ values were required, especially for the case of lighter projectiles. In the case of ${ }^{12} \mathrm{C}+{ }^{232} \mathrm{Th}$, no satisfactory $r_{0}$ value could be found for $a=1.2 \mathrm{fm}$ that did not exhibit signs of reflected flux from the potential. As a result, the maximum diffuseness that could be used in this case was $a=1.08 \mathrm{fm}$.

\section{Nuclear structure parameters}

While the Woods-Saxon potential parameters play a strong role in the overall evolution of the calculated fusion cross sections as a function of energy, the nuclear structure of the target and projectile nuclei play a more subtle role in shaping cross sections at and below the barrier, as well as $\ell$ distributions above the barrier. In CCFULL, the collective structure of these nuclei is included in order to take this subtle influence into account.

Because experimental barrier distributions were not available for all reactions, a rigorous coupled-channels calculation was not carried out. However, in this work, we have found that nuclear structure parameters, while important for completely accurate $\ell$ distributions, generally have a small effect on the final TSM fission anisotropies. With this in mind, the procedure used for selecting which structural parameters to include was guided by past experience and available data. This procedure is outlined below.

For target nuclei, the type of phonon model (vibrational, rotational, anharmonic vibrational) used in the calculation was selected based on the experimental $R_{4 / 2} \equiv E\left(4_{1}^{+}\right) / E\left(2_{1}^{+}\right)$ value. In collective nuclei, $R_{4 / 2} \sim 2$ corresponds to a vibrator, $R_{4 / 2} \sim 3.33$ to a rigid rotor, and $R_{4 / 2} \sim 2.5$ to an anharmonic vibrator [47]. Nuclei with values in between these limits were assigned a structure based on the closest $R_{4 / 2}$ value and an assessment of the known level scheme [48]. Projectile nuclei can only be treated as vibrational or rotational within the model. With the exception of ${ }^{12} \mathrm{C}$, all had $R_{4 / 2}$ values and level schemes that were best represented as vibrational. Because ${ }^{12} \mathrm{C}$ has very high-lying collective excited states, it was treated as inert in the calculations [49], meaning that no structural information for this projectile was taken into account.

For target nuclei, both quadrupole and octupole phonon excitations were included to at least the two-phonon level, depending on the excitation energies of the low-lying collective nuclear states. Hexadecapole deformations (all small) were also included where possible. Only the first phonon excitation was included for projectile nuclei, where appropriate. Phonon excitation energies and deformation parameters $\left(\beta_{2,3,4}\right)$ were obtained from Refs. [50,51] or were calculated from measured $B(E \lambda)$ values [48].

In Fig. 2(a), cross sections from a sample CCFULL calculation including nuclear structure parameters is compared to

TABLE II. Woods-Saxon (WS) potential parameters used in CCFULL calculations for all reactions. Each row corresponds to the parameters used for one set of calculations; for each reaction, sets of calculations were performed to check the influence of varying the diffuseness parameter (see text). For each reaction, the average barrier energy $V_{B}$ and type of potential ( $\mathrm{SP}=$ Sao Paolo [41] or $\mathrm{SW}=$ Swiatecki [42]) used to select the WS parameters is also noted. Only two $\mathrm{Si}+\mathrm{Pb}$ reactions have been included here; all $\mathrm{Si}+\mathrm{Pb}$ calculations yield very similar results.

\begin{tabular}{lcccc}
\hline \hline Reaction & $V_{B}(\mathrm{MeV})$ & $V_{0}(\mathrm{MeV}) \mathrm{a}$ & $a(\mathrm{fm})$ & $r_{0}(\mathrm{fm})$ \\
\hline \multirow{3}{*}{${ }^{12} \mathrm{C}+{ }^{232} \mathrm{Th}$} & \multirow{2}{*}{$62.02(\mathrm{SP})$} & 190 & 0.65 & 1.10 \\
& & 205 & 1.00 & 0.95 \\
& & 258 & 0.65 & 1.10 \\
${ }^{30} \mathrm{Si}+{ }^{206} \mathrm{~Pb}$ & \multirow{2}{*}{$128.10(\mathrm{SP})$} & 319 & 1.00 & 0.95 \\
& & 261 & 1.20 & 0.91 \\
& & 257 & 0.65 & 1.10 \\
${ }^{30} \mathrm{Si}+{ }^{208} \mathrm{~Pb}$ & \multirow{2}{*}{$127.87(\mathrm{SP})$} & 318 & 1.00 & 0.95 \\
& & 260 & 1.20 & 0.91 \\
${ }^{32} \mathrm{~S}+{ }^{202} \mathrm{Hg}$ & \multirow{2}{*}{$143.48(\mathrm{SP})$} & 320 & 0.65 & 1.10 \\
& & 265 & 1.00 & 0.95 \\
& & 297 & 0.65 & 0.91 \\
${ }^{48} \mathrm{Ti}+{ }^{186} \mathrm{~W}$ & $178.07(\mathrm{SW})$ & 410 & 1.00 & 0.95 \\
& & 253 & 1.20 & 0.95 \\
& & 294 & 0.65 & 1.10 \\
${ }^{64} \mathrm{Ni}+{ }^{170} \mathrm{Er}$ & $206.6(\mathrm{SW})$ & 428 & 1.00 & 0.95 \\
& & 268 & 1.20 & 1.20 \\
\hline \hline
\end{tabular}

${ }^{\mathrm{a}}$ Used for calculations with coupling included. 


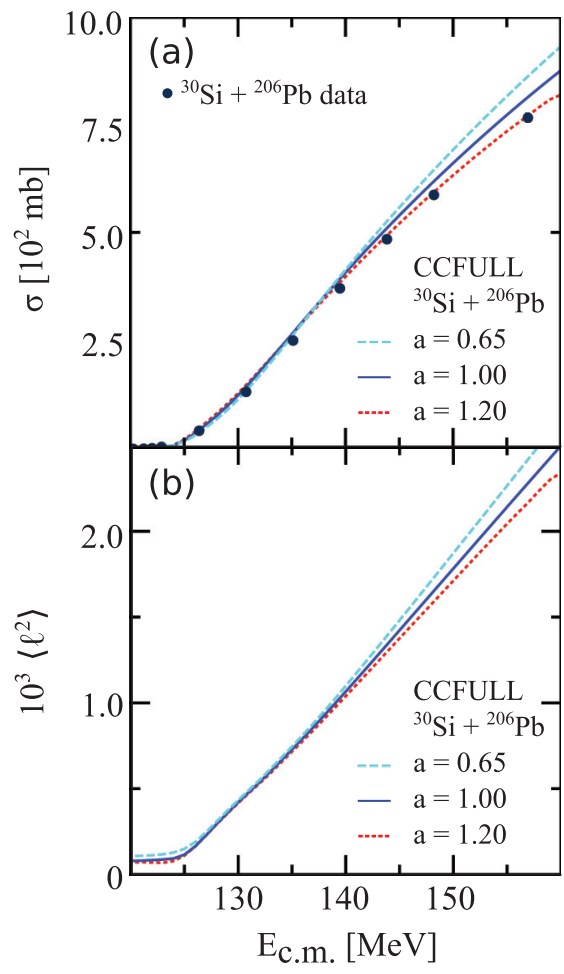

FIG. 2. (Color online) (a) Experimental fission cross sections are compared to calculated fusion cross sections for ${ }^{30} \mathrm{Si}+{ }^{206} \mathrm{~Pb}$. Calculations were performed using the code CCFULL following the procedure described and are remarkably close to observed values considering that no optimization of the Woods-Saxon potential parameters has been attempted. The evaporation-residue cross sections are expected to be negligible for this system; thus, $\sigma_{\text {cap }} \sim \sigma_{\text {fis }}$. (b) Average angular momentum squared from CCFULL corresponding to the cross-section calculations shown in panel (a). As one can see, the diffuseness parameter $a$ has a small effect on the average angular momentum squared.

data for three different diffuseness parameters. $\left\langle\ell^{2}\right\rangle$ values from the same calculation are shown in Fig. 2(b). As this example shows, the method outlined here provides reasonable agreement with data, even without detailed adjustments of potential parameters or nuclear structure inputs. Similar results were obtained for all other comparisons between calculation and data, where such comparisons were possible.

\section{B. Transition-state model}

The TSM used in this work is described in Ref. [27], and the angular anisotropy calculations were based on the code JOANNE2 [52]. The approximate expression given in Eq. (2) was not used; instead, the angular distributions were calculated for each $\ell$ value and $T$ using the angular distribution expressions provided in Ref. [53]. The saddle-point effective moment of inertia $\mathcal{J}_{\text {eff }}$ is taken from Ref. [54]; temperature $T$ is calculated assuming a level density parameter $A / 8.5 \mathrm{MeV}^{-1}$ following Tōke [55], where $A$ refers to the atomic mass of the compound nucleus. The $\ell$ distributions were calculated using CCFULL, as discussed above. Anisotropies calculated for all fission events were then compared to the experimental results. This comparison will be discussed below.

\section{RESULTS}

For all reactions studied in this work, mass ratios and MADs were measured. For reactions in which no clear evidence of quasifission was found in these two observables, angular anisotropies were also measured.

\section{A. Mass ratios}

Mass ratios, as defined in Eq. (4) and reconstructed for all FMT events for each reaction, are shown in Fig. 3 (bottom row) for selected above-barrier measurements. To understand how mass ratios evolve in each of the selected reactions, a systematic study of the widths of the mass distributions was performed.

Because mass ratios may exhibit both symmetric and asymmetric components [56], two different fits were performed. The first, a simple Gaussian fit including strength, width, and peak centroid as free parameters, assumes that the fission fragments split symmetrically (on average). The red functions plotted in Fig. 3 (bottom row) are representative of the results obtained from the first fit. As one can see, the width of the Gaussian increases with projectile charge (from left to right) until the mass ratio reaches a minimum at symmetry for the ${ }^{64} \mathrm{Ni}+{ }^{170} \mathrm{Er}$ reaction.

In order to investigate the possible presence of a massasymmetric fission component, the second fit consisted of two separate Gaussians of equal width and area, equidistant from the centroid of the Gaussian obtained from the first fit. The blue functions shown in Fig. 3 are representative of those obtained using this method; results are not shown in cases where the fit results were either identical to or did not converge to a single Gaussian. While the reactions in question may lead to both symmetric and asymmetric mass splits, requiring a third Gaussian for rigorous treatment, the relative heights and widths of the symmetric and asymmetric components are not constrained by current models. Therefore, this method is simply a tool for seeking out the potential presence of a mass-asymmetric fission mode, which is generally associated with shell effects on the potential-energy surface, and is therefore indicative of slow compound-nucleus fission after neutron emission.

\section{Single-Gaussian fit}

The mass distributions are characterized by the standard deviations of the single-Gaussian fit to the $M_{R}$ spectra. These values are shown as a function of excitation energy $E^{*}$ in Fig. 4 and are tabulated in Table III. Only reactions with the heaviest projectile have not been included in the figure and table, as a Gaussian description is unsuitable in this case. The gray line labeled $\sigma_{M R}$ (ff) corresponds to the average above-barrier $\sigma_{M R}$ obtained for the ${ }^{12} \mathrm{C}+{ }^{232} \mathrm{Th}$ reaction. Outcomes of this reaction are expected to be primarily fusion-fission above the 


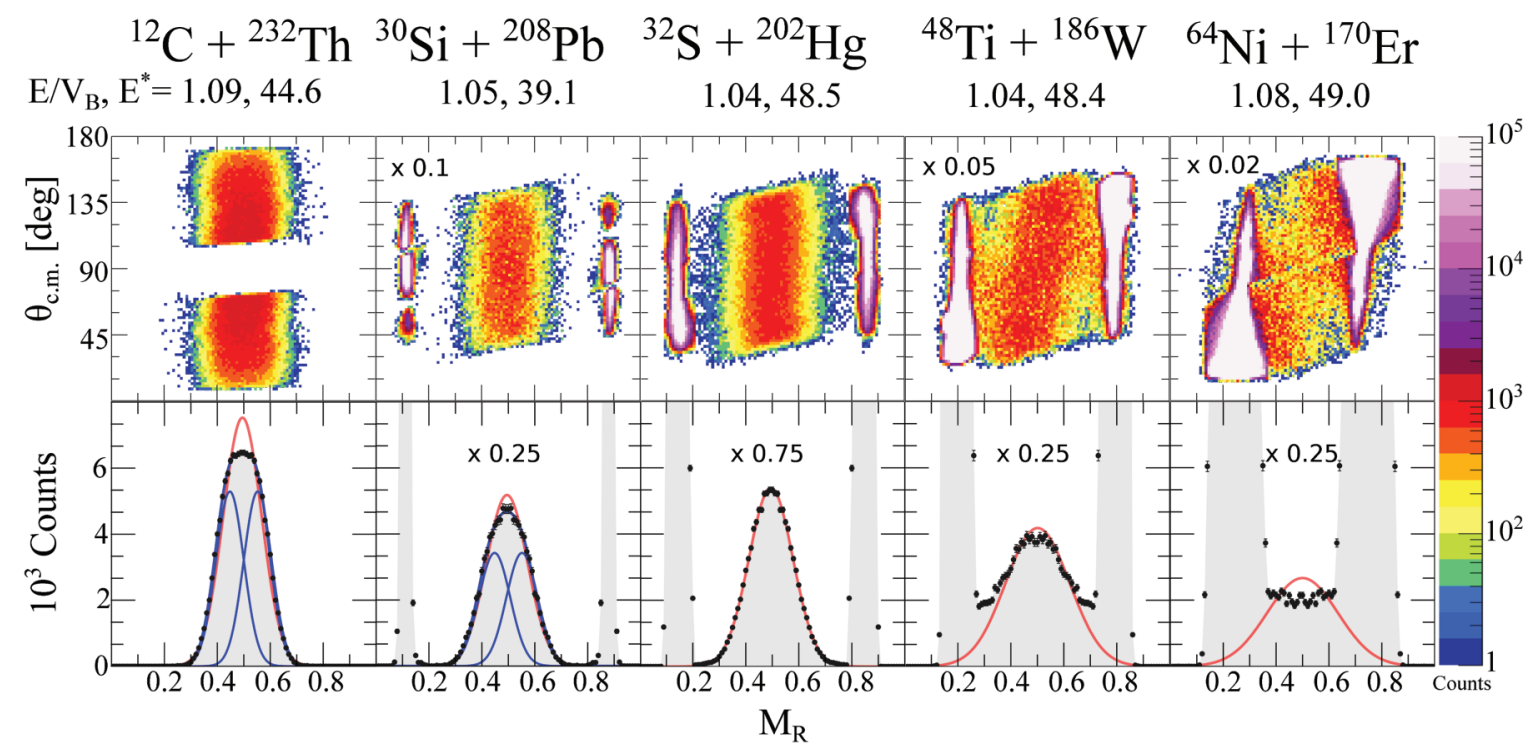

FIG. 3. (Color online) (Top row) Mass-angle distributions for each reaction are displayed for a single representative beam energy for the reactions indicated. The ratio of center-of-mass energy to average capture barrier $\left(E / V_{B}\right)$ and the compound-nucleus excitation energy $E^{*}$ (in $\mathrm{MeV}$ ) are also given. (Bottom row) Mass ratios are compared to single- or double-Gaussian fits as described in the text.

barrier, so this value is our best experimental measure of how mass ratios should behave for fusion-fission events. Any increase in the width of the mass ratio beyond this reference may then be used as evidence that other reaction mechanisms are playing a role.

As Fig. 4(a) shows, both the ${ }^{30} \mathrm{Si}+{ }^{208} \mathrm{~Pb}$ and ${ }^{32} \mathrm{~S}+{ }^{202} \mathrm{Hg}$ reactions exhibit small increases in $\sigma_{M R}$ in comparison to this reference value as a function of excitation energy. Because the variance of the mass distribution is expected to depend on $\left\langle\ell^{2}\right\rangle$, as shown in Eq. (1), interpreting these small increases requires consideration of the calculated $\left\langle\ell^{2}\right\rangle$ values shown in Fig. 4(b).

At low energies, the $\left\langle\ell^{2}\right\rangle$ values for ${ }^{12} \mathrm{C}+{ }^{232} \mathrm{Th}$ and ${ }^{32} \mathrm{~S}+$ ${ }^{202} \mathrm{Hg}$ are comparable; in this same energy region, $\sigma_{M R}$ is larger for ${ }^{32} \mathrm{~S}+{ }^{202} \mathrm{Hg}$, even when taking into account the error bars on $\sigma_{M R}$ for both reactions. This increase in $\sigma_{M R}$ in the case of ${ }^{32} \mathrm{~S}+{ }^{202} \mathrm{Hg}$ can be attributed to quasifission. At higher energies, where the $\left\langle\ell^{2}\right\rangle$ values begin to deviate for the two reactions, attributing the increase in $\sigma_{M R}$ relative to $\sigma_{M R}(\mathrm{ff})$ to quasifission is less clear.

For ${ }^{30} \mathrm{~S}+{ }^{208} \mathrm{~Pb}$, the observed increase in $\sigma_{M R}$ relative to $\sigma_{M R}(\mathrm{ff})$ is similarly inconclusive, as there is a significant difference between the $\left\langle\ell^{2}\right\rangle$ values for this reaction and ${ }^{12} \mathrm{C}+{ }^{232} \mathrm{Th}$ at all observed excitation energies. For both ${ }^{30} \mathrm{Si}+{ }^{208} \mathrm{~Pb}$ and
${ }^{32} \mathrm{~S}+{ }^{202} \mathrm{Hg}$ reactions, then, the small increase in $\sigma_{M R}$ relative to $\sigma_{M R}(\mathrm{ff})$ is difficult to interpret as a definitive signature of quasifission at all observed energies without additional measurements.

For ${ }^{48} \mathrm{Ti}+{ }^{186} \mathrm{~W}$, however, the deviation from $\sigma_{M R}(\mathrm{ff})$ is significant. Given that this reaction and ${ }^{30} \mathrm{Si}+{ }^{208} \mathrm{~Pb}$ have essentially the same $\left\langle\ell^{2}\right\rangle$ values for the energy range of interest, it is reasonable to attribute this large increase in $\sigma_{M R}$ relative to $\sigma_{M R}$ (ff) to quasifission. In the case of ${ }^{64} \mathrm{Ni}+{ }^{170} \mathrm{Er}$, the mass distribution does not even show a peak at symmetry. For the heaviest two projectiles, mass ratios are already a dramatic sign that quasifission is the dominant reaction outcome. The reason the mass ratios for reactions using the heaviest two projectiles deviate so much from the relatively narrow and smooth evolution of $\sigma_{M R}$ observed otherwise will be discussed in section $\mathrm{B}$ below.

\section{Double-Gaussian fit}

As one can see in Fig. 3 (bottom row), the double-Gaussian fit is only required to fit the $M_{R}$ spectra for reactions with the lightest two projectiles. The fit parameters for all relevant reactions are given in Table IV. For ${ }^{12} \mathrm{C}+{ }^{232} \mathrm{Th}$ reactions, the

TABLE III. Mass ratio widths obtained from single-Gaussian fits, as discussed in the text.

\begin{tabular}{|c|c|c|c|c|c|c|c|}
\hline \multicolumn{2}{|c|}{${ }^{12} \mathrm{C}+{ }^{232} \mathrm{Th}$} & \multicolumn{2}{|c|}{${ }^{30} \mathrm{Si}+{ }^{208} \mathrm{~Pb}$} & \multicolumn{2}{|c|}{${ }^{32} \mathrm{~S}+{ }^{202} \mathrm{Hg}$} & \multicolumn{2}{|c|}{${ }^{48} \mathrm{Ti}+{ }^{186} \mathrm{~W}$} \\
\hline 35.5 & $0.071(2)$ & 39.1 & $0.077(3)$ & 35.9 & $0.075(2)$ & 36.5 & $0.24(2)$ \\
\hline 44.6 & $0.072(2)$ & 49.8 & $0.086(2)$ & 39.8 & $0.079(1)$ & 39.7 & $0.20(1)$ \\
\hline 48.9 & $0.071(1)$ & & & 48.5 & 0.082 (1) & 42.8 & $0.20(1)$ \\
\hline 54.6 & $0.075(1)$ & & & 56.3 & 0.087 (1) & 48.4 & $0.16(1)$ \\
\hline
\end{tabular}




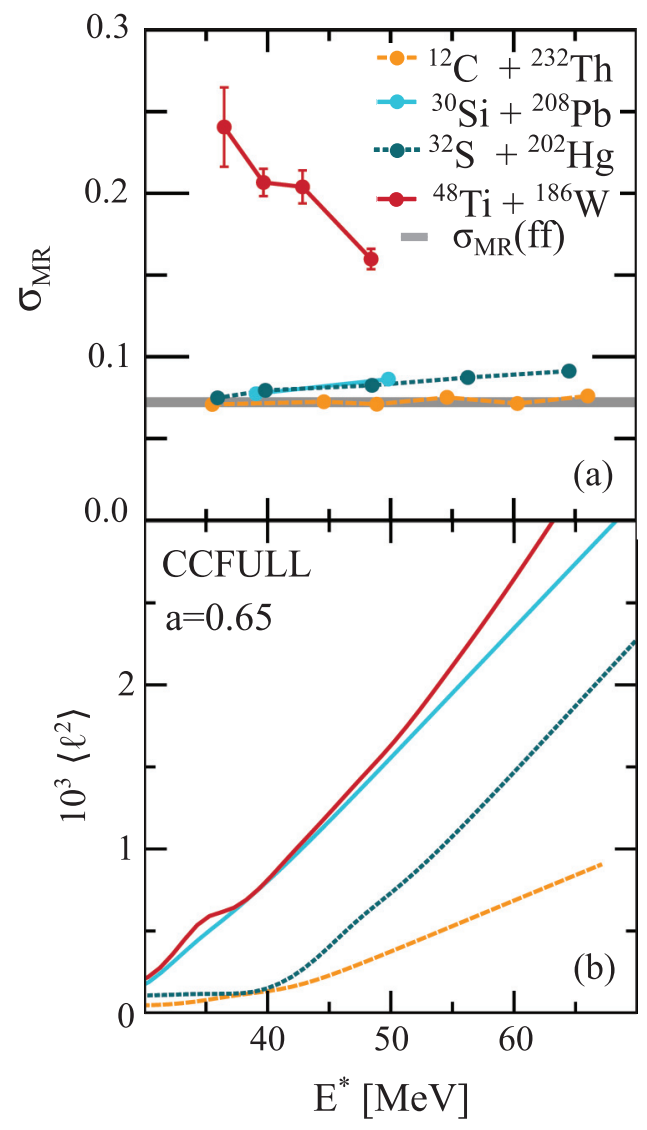

FIG. 4. (Color online) (a) $\sigma_{M R}$ values from the single-Gaussian fit plotted versus excitation energy $E^{*}$ for all reactions where such a fit was possible. In all but the reaction with the lightest projectile $\left({ }^{12} \mathrm{C}+{ }^{232} \mathrm{Th}\right)$, the fit range was narrowed to $0.27 \leqslant m_{R} \leqslant 0.73$ in order to exclude the elastics regions from the fit. In this portion of the figure, the lines connecting the data points are included to guide the eye. (b) $\left\langle\ell^{2}\right\rangle$ values calculated using CCFULL and CCDEGEN for a diffuseness parameter of $a=0.65$. The color scheme is consistent with that used for the data in the top figure.

double-Gaussian fit is best for all energies, with the asymmetric peaks growing wider as a function of excitation energy. For ${ }^{30} \mathrm{Si}+{ }^{208} \mathrm{~Pb}$, the same behavior is observed. For ${ }^{32} \mathrm{~S}+{ }^{202} \mathrm{Hg}$, the double-Gaussian fit was indistinguishable from the singleGaussian fit for the lower energies, while for the two higher energies, the double-Gaussian fit would not converge. As such, no fit results for the ${ }^{32} \mathrm{~S}+{ }^{202} \mathrm{Hg}$ reaction have been included in Table IV.

\section{B. Mass-angle distributions}

A selection of MADs resulting from this series of experiments is shown in Fig. 3 (top row). All of the MADs in Fig. 3 are for above-barrier energies; all are representative of the MADs observed for below and above-barrier energies for each reaction.

As one can see from Fig. 3 (top row), the reactions with the lightest three projectiles $\left({ }^{12} \mathrm{C},{ }^{30} \mathrm{Si}\right.$, and $\left.{ }^{32} \mathrm{~S}\right)$ show no evidence of a mass-angle correlation in their MAD plots. In contrast, reactions with the two heaviest projectiles $\left({ }^{48} \mathrm{Ti}\right.$ and $\left.{ }^{64} \mathrm{Ni}\right)$ show a significant mass-angle correlation. This mass-angle
TABLE IV. Mass-ratio widths obtained from double-Gaussian fits, where applicable, as discussed in the text. In this case, $\sigma_{M R}$ refers to the width of either peak (peak widths and areas are set to be equal in the fit), and $d_{M R}$ refers to the separation between the two peaks.

\begin{tabular}{lccccccc}
\hline \hline \multicolumn{3}{c}{${ }^{12} \mathrm{C}+{ }^{232} \mathrm{Th}$} & & \multicolumn{3}{c}{${ }^{30} \mathrm{Si}+{ }^{208} \mathrm{~Pb}$} \\
\cline { 1 - 3 } \cline { 5 - 6 }$E^{*}(\mathrm{MeV})$ & $\sigma_{M R}$ & $d_{M R}$ & & $E^{*}(\mathrm{MeV})$ & $\sigma_{M R}$ & $d_{M R}$ \\
\hline 35.5 & $0.049(1)$ & $0.051(2)$ & & 39.1 & $0.059(2)$ & $0.052(2)$ \\
44.6 & $0.053(2)$ & $0.052(2)$ & & 49.8 & $0.068(2)$ & $0.053(3)$ \\
48.9 & $0.052(2)$ & $0.049(2)$ & & & \\
54.6 & $0.056(2)$ & $0.052(2)$ & & & \\
60.3 & $0.055(2)$ & $0.046(2)$ & & & \\
66.0 & $0.060(2)$ & $0.048(2)$ & & & \\
\hline \hline
\end{tabular}

correlation demonstrates that the short reaction timescale is the primary cause of the significant change in mass ratios for reactions with the heaviest two projectiles, as presented above. Without the CUBE detector's large solid angle coverage, both the mass ratios (projected over all $\theta$ ) and the MADs would provide a less compelling case for the presence of quasifission in any of these reactions. For the reactions using the heaviest two projectiles, the MAD plots are enough to confirm that the reactions are dominated by fast quasifission occurring on average in less than a single rotation time.

\section{Angular anisotropies}

There exists no mechanism in the TSM to account for the presence of a mass-angle correlation, meaning that the TSM will certainly no longer be applicable once such a correlation is observed. This is not to say that angular anisotropies do not carry useful information on quasifission. At sufficiently small center-of-mass angles, the angular distribution is sensitive to the mean square of the angular projection $K$ onto the fission axis [58] and, therefore, can provide a direct measure of the dynamical evolution of the tilting mode in quasifission [59]. However, such measurements are beyond the scope of this work. Thus, anisotropies were only extracted from current datasets or obtained from previous measurements $[37,53,57]$ for the three reactions without a mass-angle correlation in their MADs.

For experiments conducted at the ANU, angular anisotropies were taken from measurements in which CUBE was in the $\theta=135^{\circ}$ configuration illustrated in Fig. 1(a). These resulting anisotropies are shown in Fig. 5, along with anisotropies from the TSM model calculations discussed above. In all three reactions, TSM calculations and observations of angular anisotropies for reactions using ${ }^{12} \mathrm{C},{ }^{28,30} \mathrm{Si}$, and ${ }^{32} \mathrm{~S}$ projectiles do not agree, indicating the presence of quasifission.

Results obtained using all three diffuseness parameters have been included to show that even significant changes in the parameters used for these calculations cannot yield agreement between the TSM and observation, indicating strongly that the fusion-fission picture is not adequate for these reactions. 

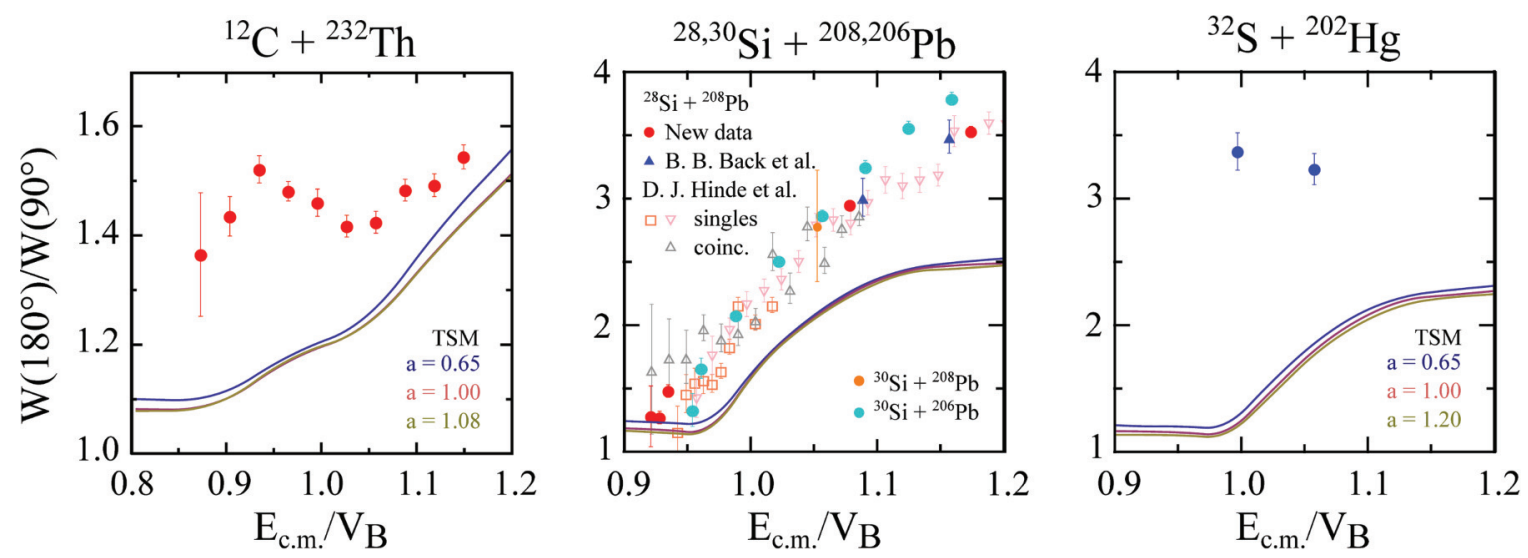

FIG. 5. (Color online) Observed and calculated anisotropies are shown for reactions using the lightest three projectiles. The angular anisotropy data presented for ${ }^{12} \mathrm{C}+{ }^{232} \mathrm{Th}$ are from Ref. [37]; data presented for ${ }^{28} \mathrm{Si}+{ }^{208} \mathrm{~Pb}$ have been taken from Refs. [53,57]. All other data are from this work. The TSM calculation results (presented as solid lines) are shown for calculations using three different diffuseness parameters, as discussed in Sec. IV. For the middle panel, TSM calculations are for ${ }^{30} \mathrm{Si}+{ }^{206} \mathrm{~Pb}$, using the same color scheme for the diffuseness parameters as shown in the legend for the panel to the right. In all three cases, the TSM is inconsistent with experiment.

Specific trends in the anisotropies for each of the three reactions will be discussed below.

\section{DISCUSSION}

As the results above illustrate, all five reactions presented in this work indicated evidence of quasifission in at least one of the fission fragment observables. Table $\mathrm{V}$ provides a summary of which signatures(s) exhibited signs of quasifission for each reaction.

The clearest and most model-independent indication of the presence of quasifission was found in the MADs, where quasifission occurring on short time scales (less than one rotation after capture) can be inferred from the presence of a correlation between mass ratio and $\theta_{\text {c.m. }}$. Such a clear correlation can only be observed if quasifission is a highly probable reaction outcome. Using a simple model [8] and the methods described in Ref. [9], time scales for the ${ }^{48} \mathrm{Ti}+{ }^{186} \mathrm{~W}$ and ${ }^{64} \mathrm{Ni}+{ }^{170} \mathrm{Er}$ reactions may be extracted. As with the very similar reactions presented in Ref. [9], timescales to scission for reactions with ${ }^{48} \mathrm{Ti}$ were longer $\left(\sim 10 \times 10^{-21} \mathrm{~s}\right)$ than those for reactions with ${ }^{64} \mathrm{Ni}\left(\sim 5 \times 10^{-21} \mathrm{~s}\right)$.

In the event that a mass-angle correlation is not observed, additional observations are required to rule out the presence of quasifission. This was made clear in our results for the three reactions that did not exhibit a mass-angle correlation. In these cases, angular anisotropies provided the clearest confirmation that quasifission is an important reaction outcome. As discussed below, the anisotropies for each reaction, which include components from both fusion-fission and quasifission, reveal that entrance channel parameters-in particular, nuclear structure-play a far more complicated role in quasifission outcomes than was once expected.

In all three of the reactions with lighter projectiles, the structure of the target nucleus is different. ${ }^{232} \mathrm{Th}$ is rotational, ${ }^{208} \mathrm{~Pb}$ is doubly magic (and best described as spherical), and ${ }^{202} \mathrm{Hg}$ is an axially asymmetric rotor. In all three cases, the anisotropies evolve very differently as a function of projectile energy.
Beginning with ${ }^{12} \mathrm{C}+{ }^{232} \mathrm{Th}$, the structural effect first explained by Hinde et al. [24,36] is observed in the large anisotropies below the barrier. As shown in Fig. 5(a), the anisotropies then begin to approach the TSM results above the barrier. The increased anisotropy below the barrier results from the reduction in the effective barrier for collisions in which the projectile is aligned with the long axis of the rotational target nucleus. Due to this alignment, the projectile-target system is elongated, making compound-nucleus formation less likely. As the projectile energy increases and other (more compact) projectile-target alignments also contribute to capture, the fraction of fusion-fission becomes larger and angular anisotropies become closer to TSM predictions; at $E_{\text {c.m. }} / V_{B} \sim 1.06, A_{\text {ratio }} \equiv(A-1)_{\text {expt. }} /(A-1)_{\text {TSM }} \sim 1.4$.

In ${ }^{28,30} \mathrm{Si}+{ }^{206,208} \mathrm{~Pb}$, as shown in Fig. $5(\mathrm{~b})$, anisotropies evolve smoothly and steadily as a function of projectile energy and are always above the TSM predictions; at $E_{\text {c.m. }} / V_{B} \sim$ 1.06 , for example, $A_{\text {ratio }} \sim 1.7$. Because these collisions are between a massive spherical nucleus and a nucleus that essentially behaves like a vibrator in the reaction, all configurations are expected to have roughly the same compactness for all energies [57]. Therefore, the smooth evolution of the anisotropies in this case indicates that quasifission contributes for all energies where capture is possible. Interestingly, though, deviations between the TSM and measurement grow as a function of projectile energy. This suggests that the probability of quasifission may in fact be enhanced for the higher angular momenta, which are only populated at the higher bombarding energies.

For the last reaction, ${ }^{32} \mathrm{~S}+{ }^{202} \mathrm{Hg}$, the data shown in Fig. 5 is currently insufficient to draw firm conclusions beyond the clear disagreement between TSM calculations and observation; at $E_{\text {c.m. }} / V_{B} \sim 1.06, A_{\text {ratio }} \sim 2.6$. It is nevertheless interesting that the measured anisotropies do not change significantly as a function of energy and in fact are at a maximum below the barrier. Looking at more complete anisotropy measurements for a reaction with somewhat similar projectile-target combinations, Fig. 3 in Ref. [60] presents a similarly flat trend for ${ }^{34} S+{ }^{168} \mathrm{Er}$. It is possible that this flattening may be due to a small decrease 
TABLE V. Summary of observables indicating the presence of quasifission for all reactions and qualitative conclusions regarding quasifission characteristics for each reaction. A $\checkmark$ indicates that the signature has been observed in the reaction in question, a $\mathrm{X}$ indicates that the signature has not been observed, and a ? indicates that no definitive conclusions could be drawn from the available evidence.

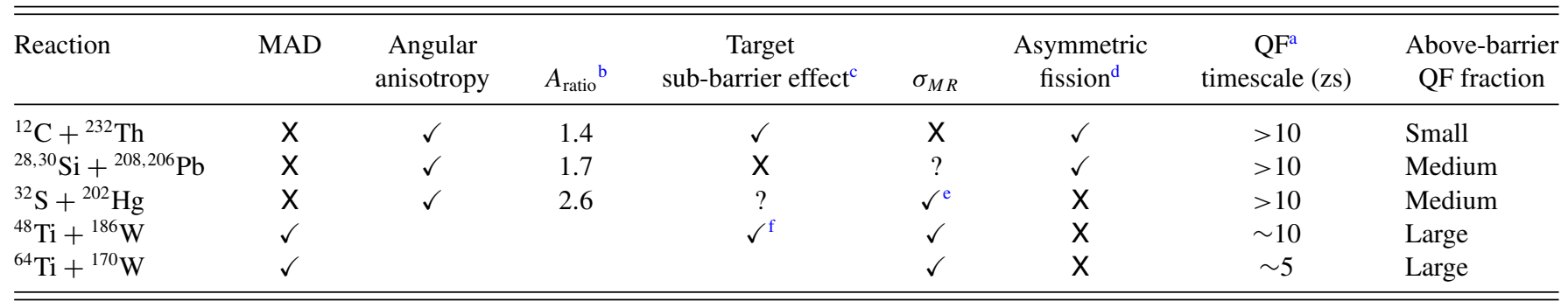

${ }^{\mathrm{a}} \mathrm{QF}=$ quasifission.

${ }^{\mathrm{b}}$ At $E_{\text {c.m. }} / V_{B} \sim 1.06$.

${ }^{\mathrm{c}}$ Due to deformation alignment, as described in Ref. 36.

${ }^{\mathrm{d}}$ As determined by improved mass-ratio fit with two Gaussians.

${ }^{\mathrm{e}}$ At least in the lower-energy data.

${ }^{\mathrm{f}}$ In $\sigma_{M R}$.

in quasifission probability as compact collisions become more favorable; however, more data is necessary before any detailed conclusion can be made.

Because fusion-fission and quasifission are probably both significant outcomes for each of these reactions, the anisotropies are shaped by the balance between these two processes as a function of projectile energy. While simple arguments of how this balance might evolve as a function of energy can be made, it is clear that the confirmation of these interpretations will continue to be difficult until a dynamical model capable of reproducing these dynamics is created.

\section{CONCLUSIONS}

We cannot separate out fusion-fission and quasifission event by event, nor can we quantitatively determine how much of each process contributes to the reaction outcomes presented here. What we can say is this: even for reactions with the lightest projectile, quasifission is clearly an important reaction outcome.

Several different signatures of quasifission have been studied for a range of reactions leading to similar or identical isotopes of curium. Despite the extreme range of projectiletarget choices presented here, evidence of quasifission has been found in one or more easily detectable observables, all of which will provide useful new benchmarks for future dynamical models. The only piece of the picture still missing in this work are evaporation-residue (ER) cross sections. But like many reactions leading to nuclei in this mass region, ER measurements for ${ }^{30} \mathrm{Si}+{ }^{208} \mathrm{~Pb}$ have already been shown to present a particular experimental challenge [61], which suggests that measurements for the other reactions discussed here would be similarly difficult.

More generally, this work has shown that the observable offering the clearest evidence for quasifission differs depending on the reaction timescale. For reactions without evidence for fast quasifission in the MAD, or where quasifission is not the dominant outcome, a comparison between angular anisotropies and TSM calculations provide the clearest signature of quasifission. This was seen in data from the ${ }^{12} \mathrm{C}+$
${ }^{232} \mathrm{Th},{ }^{28,30} \mathrm{Si}+{ }^{208,206} \mathrm{~Pb}$, and ${ }^{32} \mathrm{~S}+{ }^{202} \mathrm{Hg}$ reactions. We also see the deviations from the expectations for fusion-fission increasing with the mass of the projectile. For each of these reactions, mass ratios provided the only alternative signature. This signature, although consistent with the conclusions drawn from the angular anisotropy data, was weak. Knowledge of how the fusion-fission mass width depends on angular momentum is required before mass ratios can be used to confirm the presence of quasifission.

For reactions dominated by fast quasifission, mass ratios were a suitable and much clearer signature than for the slower reactions. However, MADs were a superior signature to study for such reactions for two reasons: first, the presence of a mass-angle correlation is a clear and nearly model-independent signature of quasifission and, second, information on the reaction timescale can be extracted from MADs as was discussed for the ${ }^{48} \mathrm{Ti}+{ }^{186} \mathrm{~W}$ and ${ }^{64} \mathrm{Ni}+{ }^{170} \mathrm{Er}$ reactions.

None of the signatures in question are currently capable of providing quantitative information on the fraction of quasifission events relative to fusion-fission events observed in a given reaction. Because quasifission and fusion-fission events cannot be separated using experimental methods, only a dynamical model of fission can provide this information.

Experimental MADs offer a motivation and testing ground for developing such a dynamical model of fusion and fission because of the reaction timescale information they provide. More importantly, MADs are a sensitive probe of the specific type of reactions currently used for superheavy-element formation and are thus a powerful tool for enhancing our ability to explore more exotic regions of the island of stability.

\section{ACKNOWLEDGMENTS}

The authors acknowledge support from Australian Research Council Grants FL110100098 and DP110102858. The authors also wish to thank J. Khuyagbaatar for a helpful discussion on the possibility of measuring evaporation-residue cross sections for similar reactions, and the Heavy Ion Accelerator Facility's accelerator and technical staff for their invaluable assistance. 
[1] G. T. Seaborg, Annu. Rev. Nucl. Sci. 18, 53 (1968).

[2] P. Armbruster, Annu. Rev. Nucl. Part. Sci. 50, 411 (2000).

[3] S. Hofmann and G. Münzenberg, Rev. Mod. Phys. 72, 733 (2000).

[4] K. Morita, K. Morimoto, D. Kaji, T. Akiyama, S. Goto, H. Haba, E. Ideguchi, K. Katori, H. Koura, H. Kudo et al., J. Phys. Soc. Jpn. 76, 043201 (2007).

[5] Yuri. Oganessian, J. Phys. G 34, R165 (2007).

[6] Yu. Ts. Oganessian, V. K. Utyonkov, Y. V. Lobanov, F. S. Abdullin, A. N. Polyakov, R. N. Sagaidak, I. V. Shirokovsky, Y. S. Tsyganov, A. Voinov, G. Gulbekian et al., Phys. Rev. C 74, 044602 (2006).

[7] V. I. Zagrebaev, A. V. Karpov, and W. Greiner, Phys. Rev. C 85, 014608 (2012).

[8] J. Tōke, B. Bock, G. X. Dai, A. Gobbi, S. Gralla, K. D. Hildenbrand, J. Kuzminski, W. Müller, A. Olmi, and H. Stelzer, Nucl. Phys. A 440, 327 (1985).

[9] R. du Rietz, D. J. Hinde, M. Dasgupta, R. G. Thomas, L. R. Gasques, M. Evers, N. Lobanov, and A. Wakhle, Phys. Rev. Lett. 106, 052701 (2011).

[10] S. Mitsuoka, H. Ikezoe, K. Nishio, K. Satou, and J. Lu, Phys. Rev. C 65, 054608 (2002).

[11] G. N. Knyazheva, E. M. Kozulin, R. N. Sagaidak, A. Y. Chizhov, M. G. Itkis, N. A. Kondratiev, V. M. Voskressensky, A. M. Stefanini, B. R. Behera, L. Corradi et al., Phys. Rev. C 75 , 064602 (2007).

[12] C. Simenel, D. J. Hinde, R. du Rietz, M. Dasgupta, M. Evers, C. J. Lin, D. H. Luong, and A. Wakhle, Phys. Lett. B 710, 607 (2012).

[13] D. J. Hinde, M. Dasgupta, and A. Mukherjee, Phys. Rev. Lett. 89, 282701 (2002).

[14] C. Lebrun, F. Hanappe, J. LeColley, F. Lefebvres, C. Ngô, J. Péter, and B. Tamain, Nucl. Phys. A 321, 207 (1979).

[15] R. Rafiei, R. G. Thomas, D. J. Hinde, M. Dasgupta, C. R. Morton, L. R. Gasques, M. L. Brown, and M. D. Rodriguez, Phys. Rev. C 77, 024606 (2008).

[16] B. B. Back, J. Phys.: Conf. Ser. 282, 012003 (2011).

[17] M. E. Faber, Phys. Rev. C 24, 1047 (1981).

[18] B. G. Glagola, B. B. Back, and R. R. Betts, Phys. Rev. C 29, 486 (1984).

[19] M. G. Itkis and A. Y. Rusanov, Phys. Part. Nucl. 29, 160 (1998).

[20] R. Bock, Y. T. Chu, M. Dakowski, A. Gobbi, E. Grosse, A. Olmi, H. Sann, D. Schwalm, U. Lynen, W. Müller et al., Nucl. Phys. A 388, 334 (1982).

[21] W. Q. Shen, J. Albinski, A. Gobbi, S. Gralla, K. D. Hildenbrand, N. Herrmann, J. Kuzminski, W. F. J. Müller, H. Stelzer, J. Tke et al., Phys. Rev. C 36, 115 (1987).

[22] V. S. Ramamurthy and S. S. Kapoor, Phys. Rev. Lett. 54, 178 (1985).

[23] B. B. Back, R. R. Betts, K. Cassidy, B. G. Glagola, J. E. Gindler, L. E. Glendenin, and B. D. Wilkins, Phys. Rev. Lett. 50, 818 (1983).

[24] D. J. Hinde, M. Dasgupta, J. R. Leigh, J. P. Lestone, J. C. Mein, C. R. Morton, J. O. Newton, and H. Timmers, Phys. Rev. Lett. 74, 1295 (1995).

[25] H. Q. Zhang, C. L. Zhang, C. J. Lin, Z. H. Liu, F. Yang, A. K. Nasirov, G. Mandaglio, M. Manganaro, and G. Giardina, Phys. Rev. C 81, 034611 (2010).

[26] R. Vandenbosch and J. R. Huizenga, Nuclear Fission (Academic, New York, 1973).
[27] C. R. Morton, D. J. Hinde, J. R. Leigh, J. P. Lestone, M. Dasgupta, J. C. Mein, J. O. Newton, and H. Timmers, Phys. Rev. C 52, 243 (1995).

[28] D. J. Hinde, D. Hilscher, H. Rossner, B. Gebauer, M. Lehmann, and M. Wilpert, Phys. Rev. C 45, 1229 (1992).

[29] J. Wilczyński, K. Siwek-Wilczyńska, and H. W. Wilschut, Phys. Rev. C 54, 325 (1996).

[30] J. Velkovska, C. R. Morton, R. L. McGrath, P. Chung, and I. Diószegi, Phys. Rev. C 59, 1506 (1999).

[31] J. U. Andersen, J. Chevallier, J. S. Forster, S. A. Karamian, C. R. Vane, J. R. Beene, A. Galindo-Uribarri, J. G. del Campo, H. F. Krause, E. Padilla-Rodal et al., Phys. Rev. Lett. 99, 162502 (2007).

[32] J. U. Andersen, J. Chevallier, J. S. Forster, S. A. Karamian, C. R. Vane, J. R. Beene, A. Galindo-Uribarri, J. G. del Campo, C. J. Gross, H. F. Krause et al., Phys. Rev. C 78, 064609 (2008).

[33] M. Morjean, D. Jacquet, J. L. Charvet, A. L'Hoir, M. Laget, M. Parlog, A. Chbihi, M. Chevallier, C. Cohen, D. Dauvergne et al., Phys. Rev. Lett. 101, 072701 (2008).

[34] M. O. Frégeau, D. Jacquet, M. Morjean, E. Bonnet, A. Chbihi, J. D. Frankland, M. F. Rivet, L. Tassan-Got, F. Dechery, A. Drouart et al., Phys. Rev. Lett. 108, 122701 (2012).

[35] D. J. Hinde, R. du Rietz, and M. Dasgupta, EPJ Web Conf. 17, 04001 (2011).

[36] D. J. Hinde, M. Dasgupta, J. R. Leigh, J. C. Mein, C. R. Morton, J. O. Newton, and H. Timmers, Phys. Rev. C 53, 1290 (1996).

[37] J. C. Mein, D. J. Hinde, M. Dasgupta, J. R. Leigh, J. O. Newton, and H. Timmers, Phys. Rev. C 55, R995 (1997).

[38] R. Butt, Ph.D. thesis, The Australian National University, 2003.

[39] K. Hagino, N. Rowley, and A. T. Kruppa, Comp. Phys. Comm. 129, 143 (1999).

[40] K. Hagino and N. Takigawa, Prog. Theor. Phys. 128, 1061 (2012).

[41] L. C. Chamon, B. V. Carlson, L. R. Gasques, D. Pereira, C. De Conti, M. A. G. Alvarez, M. S. Hussein, M. A. Cândido Ribeiro, E. S. Rossi, and C. P. Silva, Phys. Rev. C 66, 014610 (2002).

[42] W. J. Swiatecki, K. Siwek-Wilczyńska, and J. Wilczyński, Phys. Rev. C 71, 014602 (2005).

[43] J. O. Newton, R. D. Butt, M. Dasgupta, D. J. Hinde, I. I. Gontchar, C. R. Morton, and K. Hagino, Phys. Rev. C 70, 024605 (2004).

[44] L. R. Gasques, M. Evers, D. J. Hinde, M. Dasgupta, P. R. S. Gomes, R. M. Anjos, M. L. Brown, M. D. Rodríguez, R. G. Thomas, and K. Hagino, Phys. Rev. C 76, 024612 (2007).

[45] D. J. Hinde, R. L. Ahlefeldt, R. G. Thomas, K. Hagino, M. L. Brown, M. Dasgupta, M. Evers, L. R. Gasques, and M. D. Rodriguez, Phys. Rev. C 76, 014617 (2007).

[46] M. Evers, M. Dasgupta, D. J. Hinde, L. R. Gasques, M. L. Brown, R. Rafiei, and R. G. Thomas, Phys. Rev. C 78, 034614 (2008).

[47] R. F. Casten, Nuclear Structure from a Simple Perspective, 2nd ed. (Oxford University Press, New York, NY, 2000).

[48] URL http://www.nndc.bnl.gov/ensdf. 
[49] K. Hagino, N. Takigawa, M. Dasgupta, D. J. Hinde, and J. R. Leigh, Phys. Rev. Lett. 79, 2014 (1997).

[50] S. Raman, C. W. Nestor, Jr., and P. Tikkanen, At. Data Nucl. Data Tables 78, 1 (2001)

[51] T. Kibédi and R. H. Spear, At. Data Nucl. Data Tables 80, 35 (2002).

[52] J. P. Lestone, J. R. Leigh, J. O. Newton, D. J. Hinde, J. X. Wei, J. X. Chen, S. Elfström, and M. Zielinska-Pfabé, Nucl. Phys. A 559, 277 (1993).

[53] B. B. Back, R. R. Betts, J. E. Gindler, B. D. Wilkins, S. Saini, M. B. Tsang, C. K. Gelbke, W. G. Lynch, M. A. McMahan, and P. A. Baisden, Phys. Rev. C 32, 195 (1985).

[54] A. J. Sierk, Phys. Rev. C 33, 2039 (1986).

[55] J. Tōke and W. J. Światecki, Nucl. Phys. A 372, 141 (1981).

[56] D. C. Hoffman, Acc. Chem. Res. 17, 235 (1984).
[57] D. J. Hinde, C. R. Morton, M. Dasgupta, J. R. Leigh, J. C. Mein, and H. Timmers, Nucl. Phys. 592, 271 (1995).

[58] B. B. Back, P. B. Fernandez, B. G. Glagola, D. Henderson, S. Kaufman, J. G. Keller, S. J. Sanders, F. Videbæk, T. F. Wang, and B. D. Wilkins, Phys. Rev. C 53, 1734 (1996).

[59] K. Lützenkirchen, J. Kratz, G. Wirth, W. Brüchle, K. Sümmerer, R. Lucas, J. Poitou, and C. Grégoire, Nucl. Phys. A 452, 351 (1986).

[60] C. R. Morton, A. C. Berriman, R. D. Butt, M. Dasgupta, A. Godley, D. J. Hinde, and J. O. Newton, Phys. Rev. C 62, 024607 (2000).

[61] J. Khuyagbaatar, K. Nishio, S. Hofmann, D. Ackermann, S. Heinz, F. P. Hessberger, R. Mann, and B. Sulignano, in GSI Scientific Report 2007-1, 2007, http://www-alt. gsi.de/informationen/wti/library/scientificreport2006/PAPERS/ NUSTAR-SHE-04.pdf. 